\title{
Correction to: An evidence-based review of the genotoxic and reproductive effects of sulfur mustard
}

\author{
Fazlullah Khan ${ }^{1,2,3} \cdot$ Kamal Niaz $^{1,2,3} \cdot$ Fatima Ismail Hassan ${ }^{1,2,3} \cdot$ Mohammad Abdollahi $^{2,3}$
}

Received: 25 April 2018 / Accepted: 3 May 2018 / Published online: 12 June 2018

c) Springer-Verlag GmbH Germany, part of Springer Nature 2018

\section{Correction to: Arch Toxicol (2017) 91(3):1143-1156 https://doi.org/10.1007/s00204-016-1911-8}

This is with reference to the note of the Editor-in-Chief of the Archives of Toxicology, regarding similarity of Table 1 in Khan et al. (2017) to Table 1 of a paper by Marzony et al. (2016), and similarity between Figure 2 and 3 in Khan et al. (2017) to the Figure 1 and 2 in Marzony et al. (2016) and asking to explain and cite the mentioned paper. It is noted that there are limited studies on the reproductive effects of sulfur mustard (SM) exposure, so similarities in the presentation of data that are extracted from same original studies are likely. Most of such studies are done in Iran because of the war between Iran and Iraq, where Iraq's time regime used SM against Iranian veterans who regretfully still suffer from some long time effects that all addressed in a comprehensive book published by Springer International Publishing Switzerland (Balali-Mood and Abdollahi 2015). To be comprehensive, several electronic databases, such as PubMed, Scopus, Medline, Web of Science, EMBASE and Google Scholar were used to reach the relevant papers to be included in our review article. Various appropriate articles not indexed in PubMed were also considered, which fulfilled the criteria for inclusion and further recovered from Google Scholar and Google. Therefore, the total numbers

The original article can be found online at https://doi.org/10.1007/ s00204-016-1911-8.

\section{Mohammad Abdollahi}

Mohammad.Abdollahi@UToronto.Ca; Mohammad@TUMS.Ac.Ir

1 International Campus, Tehran University of Medical Sciences (IC-TUMS), Tehran, Iran

2 Toxicology and Diseases Group, The Institute of Pharmaceutical Sciences (TIPS), Tehran University of Medical Sciences (TUMS), Tehran, Iran

3 Department of Toxicology and Pharmacology, Faculty of Pharmacy, Tehran University of Medical Sciences (TUMS), Tehran 1417614411, Iran (n) of included articles in our original review paper were 103 (Fig. 1).

Taking collectively, we would like to cite Marzony et al. (2016) in our paper in demonstrating the effect of SM on the male reproductive system with the following amendments:

"In a similar study, the chronic effects of SM on the testicular system and male fertility have been carried out 20 years after exposure. As mentioned in that study, male infertility was common in $23 \%$ of patients after exposure to SM and the level of semen and quality was significantly decreased in such patients (Table 1) (Amirzargar et al. 2009; Marzony et al. 2016)".

Also, it has to be expressed that the mechanisms of action of SM's male infertility that have proper evidence so far are exactly limited to those mentioned in the Figure 2. It has been shown that male spermatozoa are more prone to oxidative stress (OS) induced by SM leading to the production of reactive oxygen species (ROS) causing a reduction in sperm quality, sperm dysfunction and hence male infertility. There are limited ways for the presentation of data when data themselves are limited. In fact, we searched various papers to illustrate the mechanism of action of SM underlying male infertility, but as the mechanism of SM with reference to OS induction is the same, therefore its presentation in a separate paper poses similarity. In the Figure 1 of Marzony et al. (2016), different mechanisms, including poly (ADP-ribose) polymerase (PARP) activation, increased ROS production, antioxidant depletion, direct interaction with macromolecules (protein, lipids, and DNA) are mentioned. Also, they have focused on the calcium transport channel, calcium releasing from the channel and high calcium levels. Similarly, in the said Figure, they indicated adenine dinucleotide (NAD) and ATP depletion. In the Figure 3 of our paper, we have highlighted the mechanisms through which SM induces OS and cell death in a very different way, such as we focused on H-protein depletion, mitochondrial dysfunction, antioxidant enzymes inhibition, nitrogen oxidative stress (NOS) and 


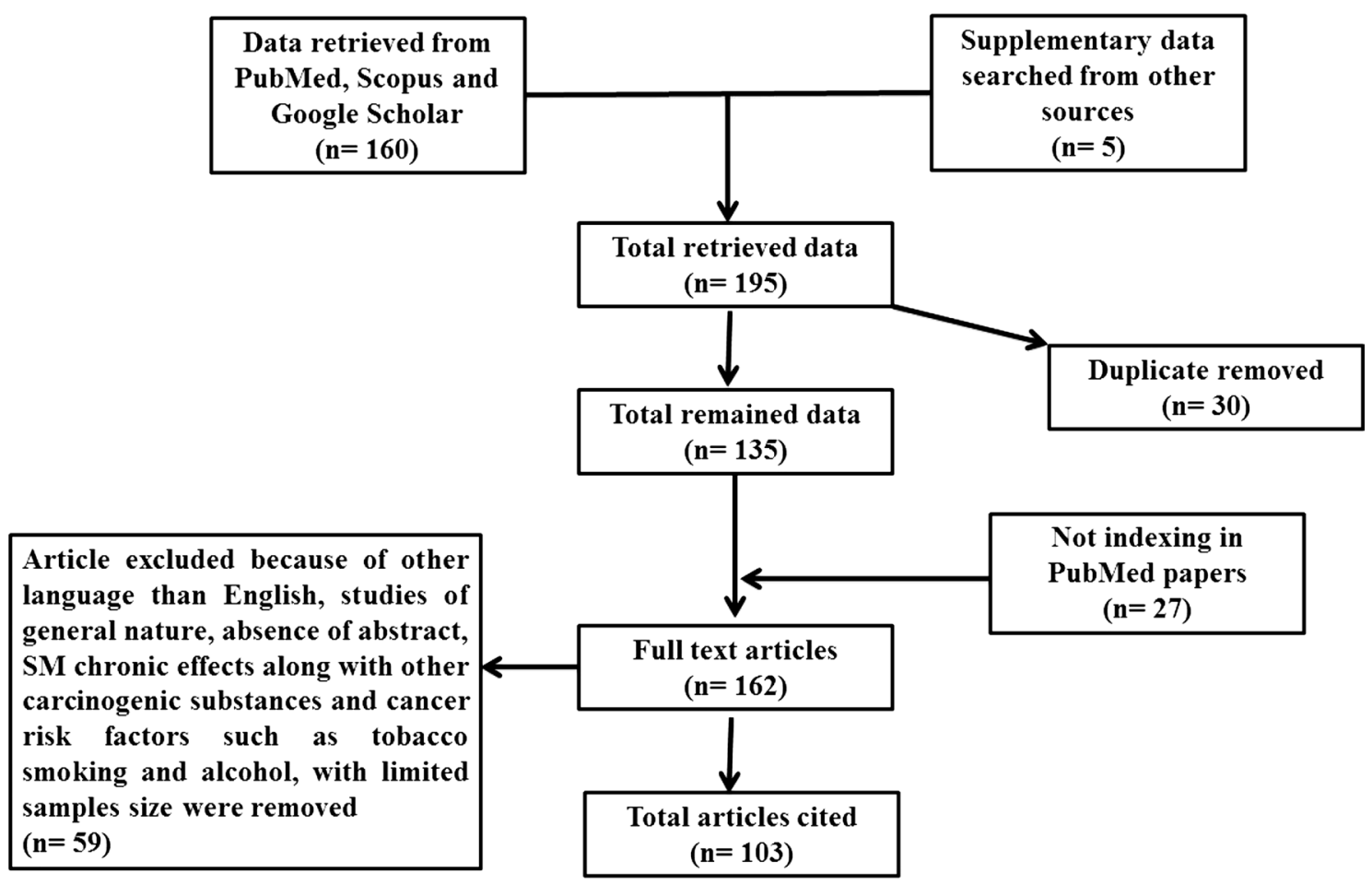

Fig. 1 The flow diagram of included studies. Adapted with permission from Springer-Verlag, GmbH, Germany, Archives of Toxicology, An evidence-based review of the genotoxic and reproductive effects of sulfur mustard, Khan et al. (2016) [license code: 4341970024760]

nitric oxide (NO) production. Similarly, we have focused on inflammatory cytokine production as a possible mechanism of cell death, such as interleukin-6 (IL-6), IL-8 and tumor necrosis factor-alpha (TNF- $\alpha$ ) and accumulation of leukocytes. Based on these grounds Figure 3 in our paper differs from Figure 1 by Marzony et al. (2016).

However, by writing this corrigendum, the authors gratefully acknowledge Marzony et al. (2016) for the Table 1 and Figure 2. The authors would like to apologize for any inconvenience caused.

\section{Compliance with ethical standards}

Conflict of interest There is no conflict of interest.

\section{References}

Amirzargar MA, Yavangi M, Rahnavardi M, Jafari M, Mohseni M (2009) Chronic mustard toxicity on the testis: a historical cohort study two decades after exposure. Int J Androl $132(4)$ :411-416

Balali-Mood M, Abdollahi M (eds) (2015) Basic and clinical toxicology of mustard compounds. Springer International Publishing, Basel, pp 1-408. https://doi.org/10.1007/978-3-31923874-6 (Print ISBN: 978-3-319-23873-9; Online ISBN: 978-3-319-23874-6)

Khan F, Niaz K, Hassan FI, Abdollahi M (2017) An evidence-based review of the genotoxic and reproductive effects of sulfur mustard. Arch Toxicol 91(3):1143-1156

Marzony ET, Ghanei M, Panahi Y (2016) Relationship of oxidative stress with male infertility in sulfur mustard-exposed injuries. Asian Pac J Reprod 5(1):1-9 